\title{
Some aspects of the epidemiology of hepatitis in Sweden
}

\author{
OLOF RINGERTZ \\ M.D.
}

Statetens Bakteriologiska Laboratorium S-105 21 Stockholm, Sweden

\section{Summary}

The incidence of hepatitis in Sweden is relatively low, but an outbreak associated with the consumption of infected oysters occurred in 1955.

Subsequently, an increasing incidence was noted among track-finders. Methods of eliminating crossinfection by traces of blood among the runners greatly reduced this incidence.

Since 1964, hepatitis associated with haemodialysis and with narcotic addiction has become common.

The changes in the epidemiological pattern during the last 20 years are discussed.

THE INCIDENCE of hepatitis in Sweden is low compared with many other countries. During the 1950s and 1960s approximately 1000 cases have been reported every year, corresponding to ten to fifteen cases per 100,000 inhabitants. In the other Scandinavian countries the figure usually varies between fifteen and twenty cases per 100,000 inhabitants. Western Germany, Ireland, and Italy in 1965 reported between thirty-five and fifty cases per 100,000 inhabitants, while Belgium and Portugal reported only seven to eight. In eastern Europe the figures are considerably higher and usually vary between two hundred to three hundred cases per 100,000 inhabitants.

The figures must be interpreted with great care as low figures may reflect a hyperendemic situation or a less reliable reporting system.

As can be seen from Fig. 1 our statistics go back to 1931 although the disease was not officially notifiable until the beginning of the $1940 \mathrm{~s}$. The peak, with a maximum of 12,000 cases a year, was however not caused by the fact that the disease was made notifiable but is believed to have its background in the considerable mobility of people during wartime, the decrease of hygiene in food handling and the use of unsuitable water-reservoirs. Many waterborne hepatitis outbreaks were reported during these years.

Immediately after the war the figures dropped to a level of approximately 1000 cases a year.

The great similarity between the clinical features of infectious hepatitis and serum hepatitis, as well as the frequently obscure and incomplete epidemiological data usually made it impossible to establish a correct differential diagnosis. However, it has been generally assumed that the majority of the cases of hepatitis during this time were infectious hepatitis.

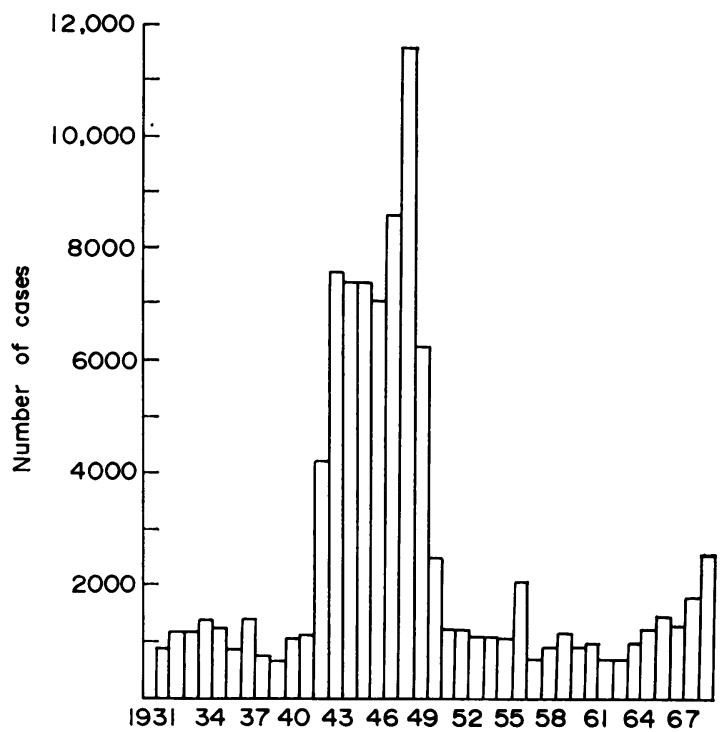

FIG. 1. Hepatitis in Sweden, 1931-69.

The fact that Sweden has had low figures and a comparatively reliable reporting system has made it possible to observe and study outbreaks of hepatitis in the population.

During the 1950s and 1960s a few hepatitis outbreaks of general interest have occurred in Sweden, and I will briefly review some of them as they do reflect the changes in the epidemiology of hepatitis that have occurred.

\section{Oyster-borne hepatitis}

Over Christmas 1955 and during the following weeks, the number of cases of hepatitis rose strikingly, especially in the cities of Gothenberg, Stockholm, and Uppsala (Roos, 1956; Zetterberg, 1964). The epidemiological investigation revealed that the first cases had been due to infection from eating oysters. It was also found that a fisherman in the little village of Havstensund on the Swedish west coast had fallen ill with hepatitis on 24 November. The sewage outlet of the village was quite close to the place where fish and oysters were kept in cages. Usually the oysters were stored for 2 or 3 days, sometimes a week before delivery. Most of the patients had eaten their oysters during the end of November or the beginning of December while the 
onset of the disease occurred approximately 1 month later (Fig. 2). For some of the patients who had eaten oysters on one occasion only it was possible to establish the exact date of consumption as well as the date of onset. As shown in Fig. 3 the length of the incubation period varied from 18-44 days with an average length of 29 days. There was no significant difference in the length of the incubation period between those who had eaten only one or two oysters and those who had eaten more than six. It could be concluded that the consumption of one single oyster was sufficient to produce hepatitis with clinical symptoms.

Attempts were also made to analyze how many of the persons who had consumed oysters became ill. Everyone who had clinical symptoms was asked about the number of persons who had eaten oysters with him at the same time. Through this analysis it was possible to establish that during the week 25 November to 1 December, the attack rate was

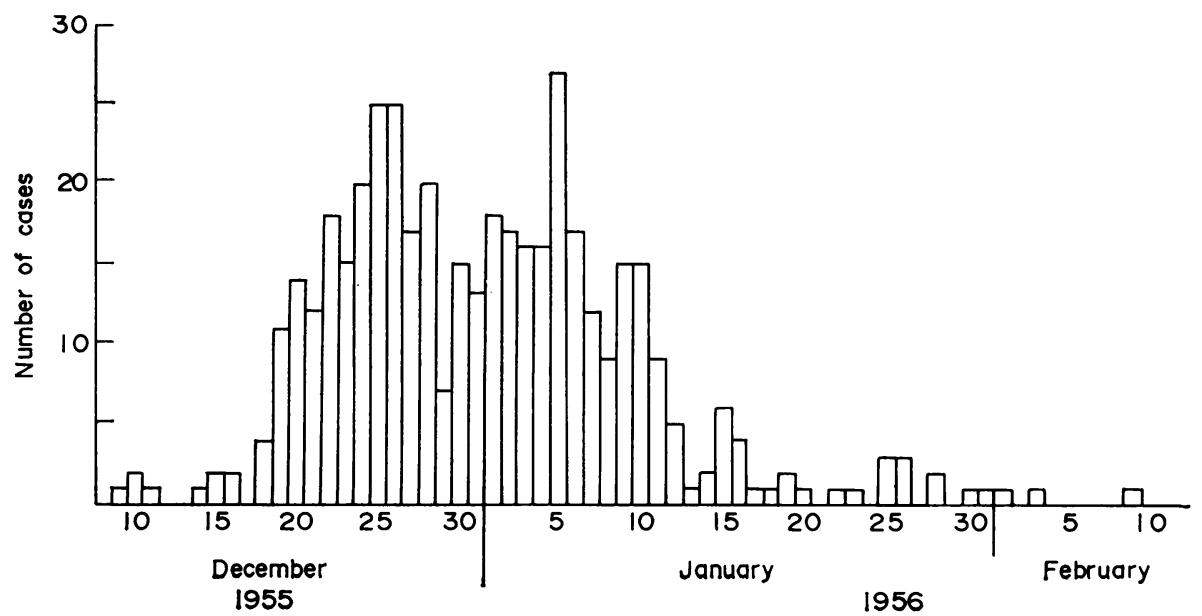

Fig. 2. Date of onset in 434 cases of hepatitis caused by contaminated oysters. (By kind permission of Professor B. Zetterberg.)

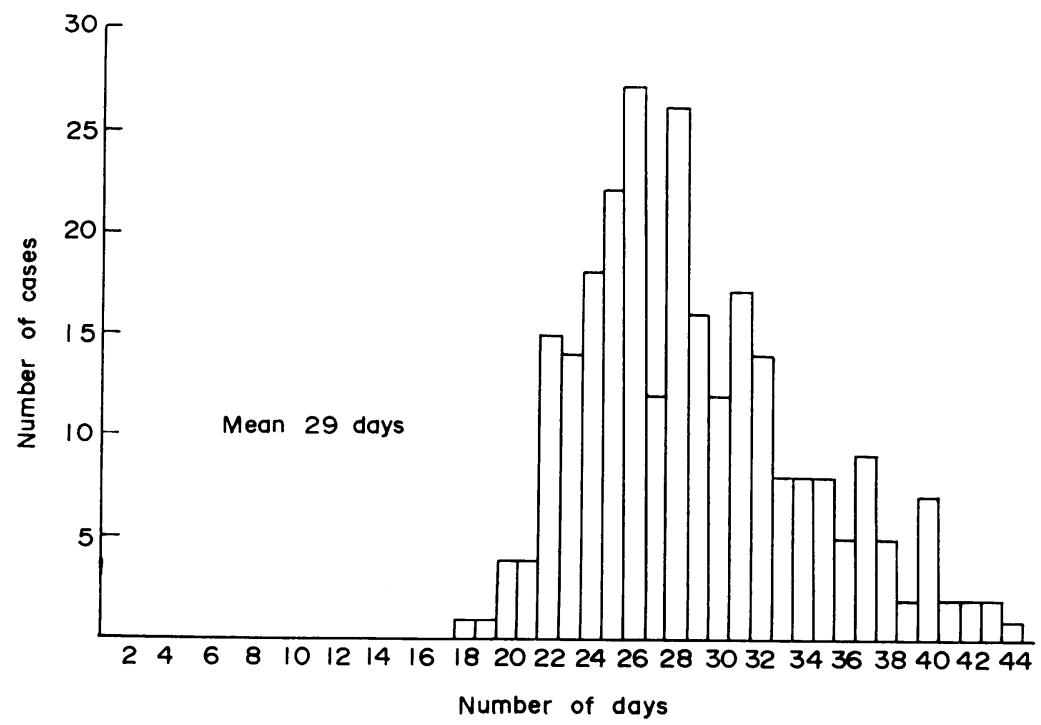

FIG. 3. Length of incubation period in 262 cases of hepatitis caused by contaminated oysters. (By kind permission of Professor B. Zetterberg.) 
approximately $75 \%$ while during the following weeks it was $60 \%, 50 \%$, and $13 \%$ respectively.

It could also be concluded that the majority of the cases belonged to the highest social groups.

After this outbreak the number of hepatitis cases was normal for a few years.

\section{Hepatitis among cross-country track-finders}

During the autumn of 1959 an increasing incidence of hepatitis was reported among Swedish track-

Cross-country track-finding is a very popular sport in Sweden. The total registered number of track-finders is approximately 25,000 and approximately 10,000 of them take an active part in trackfinding competitions.

At these competitions the competitors are given the bearings of a number of check points, approximately 1 or 2 kilometres apart. These points have to be passed in a certain order in as short a time as possible. The runners are allowed to choose, with the aid of map and compass, whatever route they prefer. The runners are divided into several different classes according to sex, age, and skill. Competitions are arranged over all types of terrain although most of them take place in woodland. For practical reasons the starting place and the goal are usually situated close together, often near a lake, a brook finders.

or a pond, to facilitate bathing or washing after the race.

A large number of competitions are arranged every year all over the country, mainly during two seasons. The spring season starts in the middle or end of March and finishes by the end of May, whereas the autumn season is from the beginning of August to the end of October. Most of the competitions take place during the autumn. The number of competitors usually varies between 200 and 400 but each season there are several competitions with more than 1000 participants.

When the epidemiological investigation was started in 1960 it was soon revealed that the first cases of hepatitis among track-finders had already occurred in western Sweden during the autumn of 1957 (Fig. 4) (Ringertz \& Zetterberg, 1967). During the following year (Fig. 5) most cases still occurred in the western part of the country. Gradually the epidemic moved east, south, and north (Fig. 6), reaching the far north areas of Sweden during 1961-62.

From the beginning of the outbreak it was obvious that the disease had a very characteristic seasonal distribution (Fig. 7). There were two peaks, one in August and one in December to January, that is approximately 4 months after the competition seasons.
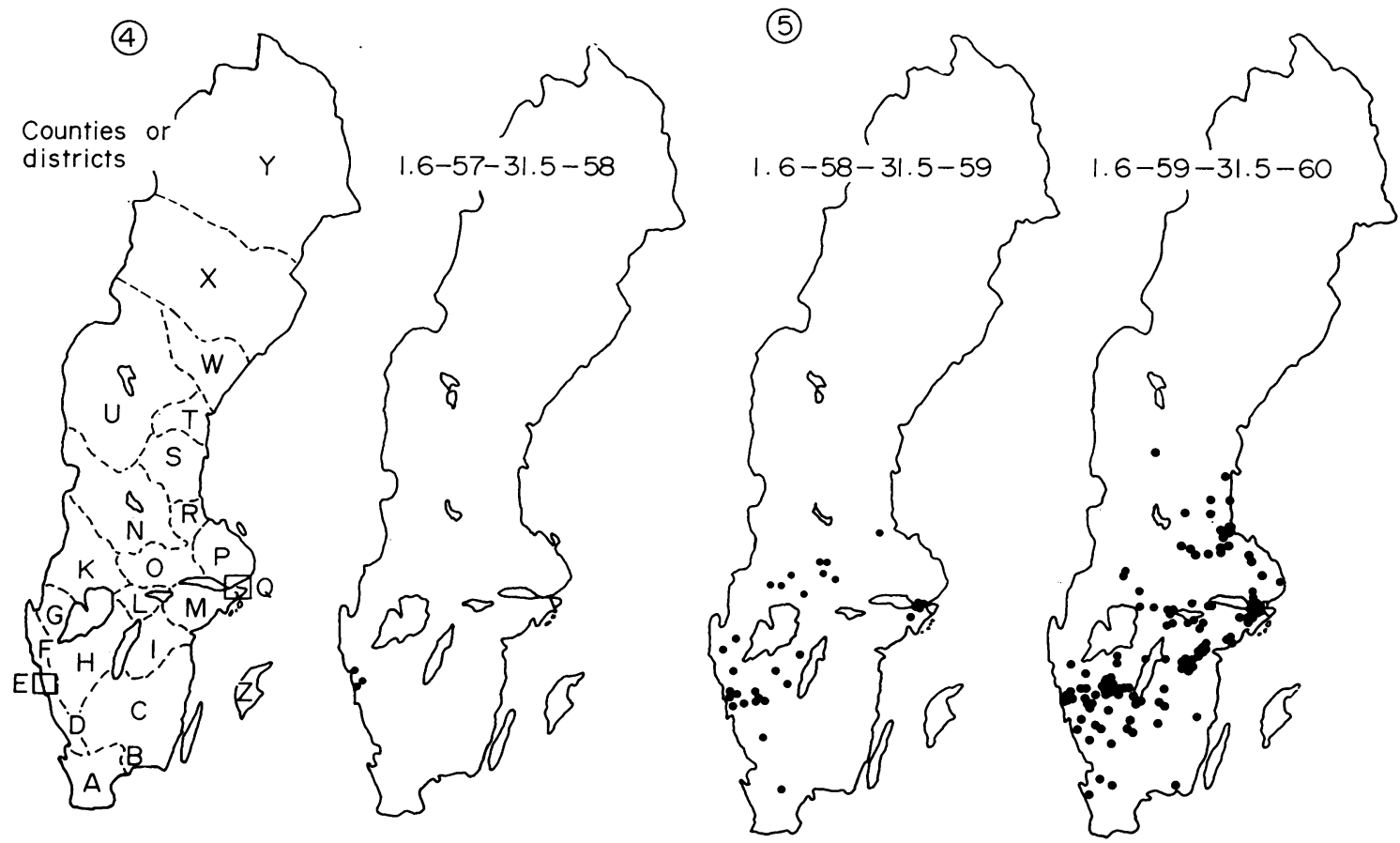

FIGs. 4 and 5. Geographic spread of hepatitis among Swedish track-finders, 1957-62. 


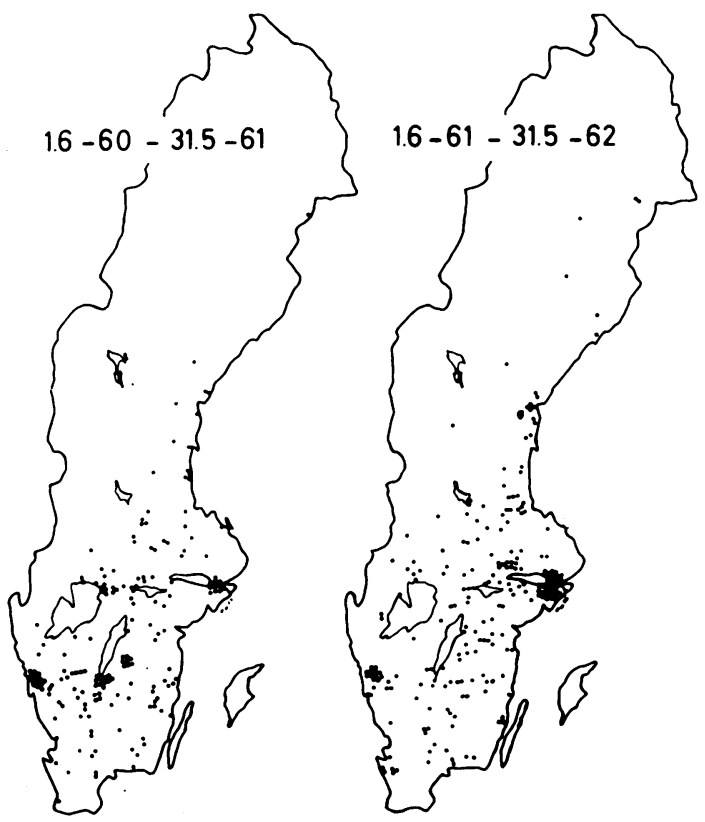

FIG. 6. Geographic spread of hepatitis among Swedish track-finders, 1960-62.
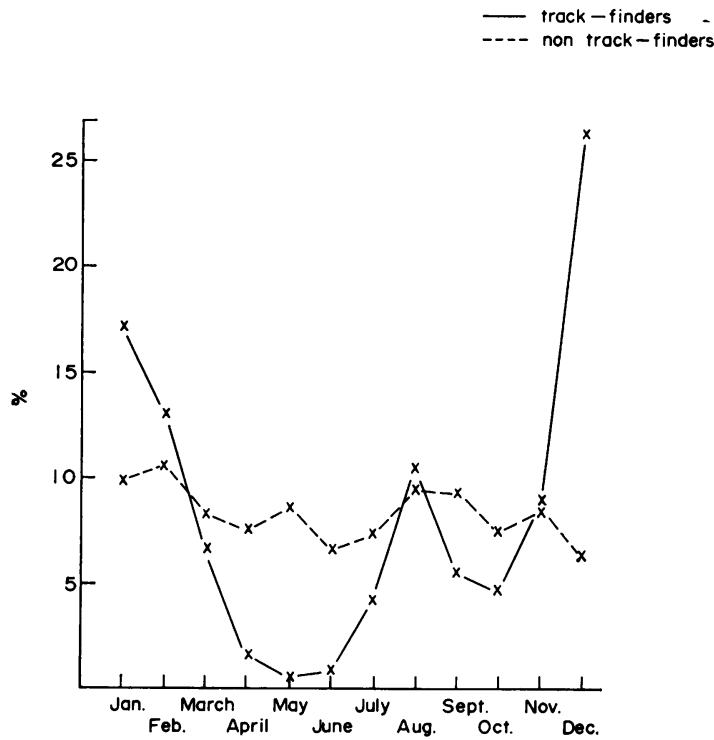

FIG. 7. Seasonal distribution of hepatitis among trackfinders, 1957-62. —_, Track-finders; - - - , non-trackfinders.

Detailed data concerning the competitions were collected and it could be established that over $50 \%$ of 4613 participations of 476 infected track-finders had taken place between 90 and 150 days before the onset of the disease. Only twelve of the 476 patients had not taken part in any competiton during this period.

It was also found that during the competitions the competitors usually sustained wounds and scratches, especially on their legs, and one third of the patients reported that during the 6 months preceding the onset of their illness they had received deep wounds.

The outbreak spread, not only geographically, but also within the different competition classes of the sport. During the first few years, the majority of the cases occurred among male track-finders between the ages of 21 and 42. Later in the outbreak the epidemic also involved women and the lower agegroups.

The arrangements for washing after the competitions were usually quite primitive. The competitors generally washed in a nearby creek or lake. Sometimes they used plastic tubs, which they filled with water and stepped into, washed their legs, poured out the water and passed the tub on to the next competitor without cleaning it, thus making it possible to transmit traces of blood to the next user. There were also other possibilities of transmitting infected blood from one track-finder to another. Close to the check points the track-finders might, for example scratch themselves on the same bushes.

The length of the incubation period, the clinicat picture and the presence of a possible mechanism for the transmission supported the diagnosis of serum hepatitis. Therefore, during the spring of 1962 all competitions were prohibited and by the time the autumn season started the hygiene had been improved and protective clothing during the competitions was compulsory; very few cases occurred during the following years (Fig. 8). The number of cases went down to the level that could be expected statistically for the group, that is, approximately three cases every year. In 1965 the track-finders' association abolished the preventive measures and during the autumn and winter another forty-one cases occurred among the track-finders. This is particularly interesting as these new cases occurred simultaneously in all parts of the country and the number of cases was of an order which at the beginning of the outbreak had taken several years to build up. This indicates that after 3 years there may still have been a number of carriers among the track-finders. The preventive measures were immediately re-introduced and the situation has been quite uneventful ever since.

When the outbreak started there was some doubt as to the nature of the disease. A thorough clinical investigation revealed that all the clinical features accorded with the diagnosis of serum hepatitis (Gille, Ringertz and Zetterberg, 1967). The disease was characterized by an insidious onset. Tempera- 


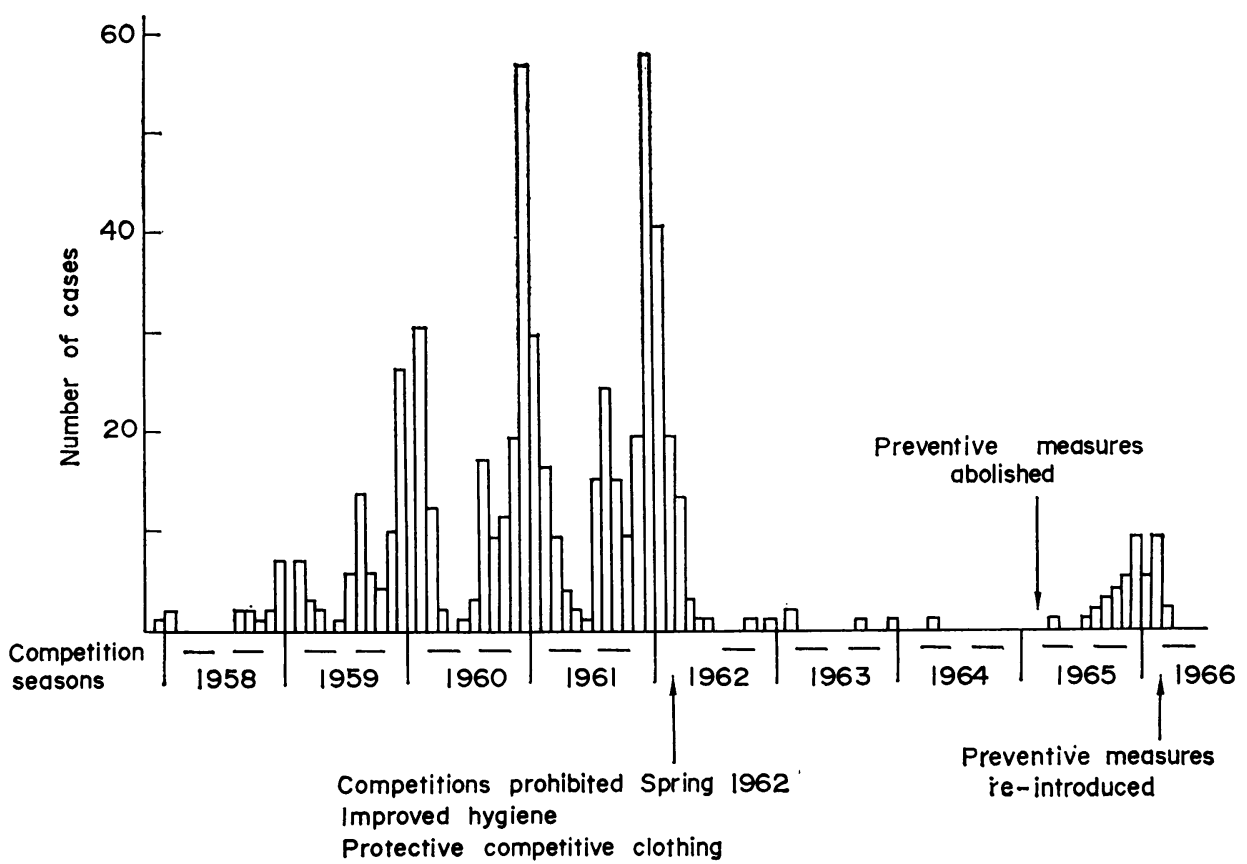

Fig. 8. Incidence of hepatitis among track-finders according to month.

tures over $38^{\circ} \mathrm{C}$ were rare, high SGOT levels were common etc. Also liver biopsies taken from some of the patients supported the diagnosis of hepatitis. The disease generally ran a mild or moderate course. One fatal case occurred.

During 1961-62, ninety-one sera from sixty-nine track-finders were collected and have been stored frozen since then. At the time we did not know what they could be used for but recently we have carried out Australia antigen tests on them. Table 1 shows that all sera taken less than 10 days after the onset of the disease were Australia antigen-positive and we find a decreasing frequency of positive sera with time after the onset. This, I think, is the final evidence that the track-finders were really suffering from serum hepatitis (Berg, Ringertz and Espmark, 1971).

TABLE 1. Frequency of Au-antigen in ninety-one sera from sixty-nine track-finders with hepatitis

\begin{tabular}{ccc}
\hline $\begin{array}{c}\text { Days after onset } \\
\text { of illness }\end{array}$ & Au +/Total & $\begin{array}{c}\text { Frequency } \\
\%\end{array}$ \\
\hline $0-9$ & $7 / 7$ & 100 \\
$10-19$ & $13 / 18$ & 72 \\
$20-29$ & $8 / 14$ & 57 \\
$30-39$ & $12 / 21$ & 57 \\
$40-49$ & $3 / 12$ & 25 \\
$50-59$ & $2 / 10$ & 20 \\
$\geqslant 60$ & $0 / 9$ & 0 \\
\hline
\end{tabular}

\section{Hảemodialysis-associated hepatitis}

During the autumn of 1964 the first outbreak of hepatitis in a haemodialysis unit in Sweden occurred at St Erik's hospital in Stockholm. Later two other hospitals in the Stockholm area were involved and by June 1967 a total of 104 cases had been recorded (Ringertz \& Nyström, 1967). Seventy-six of them occurred among personnel, and twenty-one among patients: and seven were family contacts. Since 1967 the number has increased considerably as several new haemodialysis centres have been involved and the infection continues at the old ones. The total number is now well over 200.

As this subject will be dealt with elsewhere only a few of the features, which may be characteristic of the Swedish outbreaks, will be pointed out. In those cases where it has been possible to establish the length of the incubation period it has been found to be between 60 and 100 days.

The disease has shown some distinct clinical features (Ringertz, Nyström \& Ström, 1969). The length of the prodromal period has on an average been about 2 weeks but in a few cases over a month. The period was characterized by general symptoms such as fatigue, malaise, abdominal pains etc. but it was also noticed that arthralgia was very common, occurring in $55 \%$ of the patients while pruritus and cutaneous manifestations occurred in $30 \%$ and $20 \%$, respectively (Table 2 ). The disease was also charac- 
TABle 2. Prodromal symptoms (eighty icteric cases)

\begin{tabular}{lcccc}
\hline & \multicolumn{2}{c}{$\begin{array}{c}\text { Frequency as } \\
\text { first symptom }\end{array}$} & \multicolumn{2}{c}{ Total frequency } \\
\hline Fatigue & \multicolumn{2}{c}{$(55 \%)$} & 54 & $(68 \%)$ \\
Arthralgia & 30 & $(38 \%)$ & 44 & $(55 \%)$ \\
Malaise & 17 & $(21 \%)$ & 45 & $(56 \%)$ \\
Pruritus & 14 & $(18 \%)$ & 24 & $(30 \%)$ \\
Abdominal pains & 11 & $(14 \%)$ & 25 & $(31 \%)$ \\
Cutaneous mani- & & & & \\
$\quad$ festations & 10 & $(13 \%)$ & 16 & $(20 \%)$ \\
Muscle pains & 9 & $(11 \%)$ & 17 & $(21 \%)$ \\
\hline
\end{tabular}

terized by a rise of temperature. Forty-nine per cent of the patients had a temperature of $38^{\circ} \mathrm{C}$ or more, which was considerably more than we found in the track-finders. A comparison between the haemodialysis-associated cases of hepatitis and the track-finders showed some additional interesting features. Thus, on an average the track-finders showed a much lower maximum level of thymol turbidity than the haemodialysis-associated cases. Also the frequency of secondary cases among family contacts was considerably higher in the outbreak associated with haemodialysis.

The clinical picture was generally mild or moderate. Severe cases have occurred, but none has been fatal.

Australia antigen tests have been carried out on sera from some of the patients as well as from some of the personnel. Several of the patients have been found to be carriers of Australia antigen, while the antigen has only been demonstrated for a short period of time in the personnel.
The outbreaks of hepatitis in the haemodialysis units has drawn attention to hepatitis as an occupational hazard. In 1962, the hepatitis morbidity in Stockholm was approximately $0 \cdot 2 / 1000$ but it has now increased to $0 \cdot 8$. During the same time the hepatitis morbidity for medical personnel has increased from $0 \cdot 7$ to nearly $3 / 1000$. A closer analysis of these findings shows a very high incidence among technicians and laboratory personnel, especially those working in chemical laboratories or blood donor centres while only a few cases have occurred in bacteriological laboratories (Fig. 9). During 196567 the majority of them were personnel who had been in contact with haemodialysis units. Most of them were technicians. High figures can also be noted for nurses, nurses' assistants, and physicians. Here also contact with patients subjected to haemodialysis treatment or with blood samples from them seems to have been an important mode of transmission.

Another likely source of infection for some of the personnel is the contact with narcotic addicts. The number of drug addicts with hepatitis seen at the infectious disease hospital in Stockholm has increased enormously during the last few years (Ström, unpublished data). In 1962 only eight cases were reported, while in 1967 and 1968 it was over 4008 During $196950 \%$ of all the hepatitis cases reporte in Sweden were serum hepatitis.

\section{Changes in the epidemiological patterns}

We know that increased travelling during the last 10 years has caused an increasing number of 
Salmonella infections in Sweden. We have therefore also tried to calculate the number of cases of hepatitis which may have been contracted abroad. It was found that in 1962 approximately $16 \%$ of all the hepatitis patients had visited some areas outside Scandinavia during the 2 months preceding their illness. In 1969 the corresponding figure for patients with infectious hepatitis was $12 \%$. Approximately one third of them were people who had been working abroad, such as sailors, tourist guides etc.

There have been large changes in the epidemiological patterns of hepatitis in Sweden during the last 20 years. During the 1930s and the 1940s the seasonal distribution (Fig. 10) showed a characteristic peak in the late autumn. In the 1950s the track-finders caused a peak in January and during the last decade the curve has been quite flat.

In 1962 the age distribution only had a slight peak in the age group 20-30 years, but in 1969 it showed a very marked maximum for this age group (Fig. 11). This is probably caused by the increasing number of cases among young drug addicts, but is also a result of the high hepatitis morbidity among medical personnel.

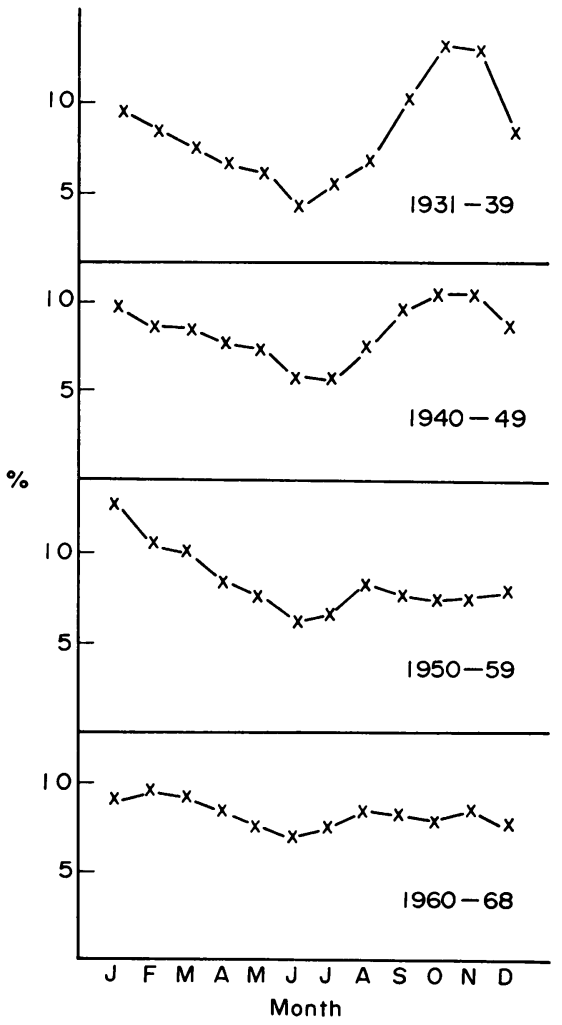

FIG. 10. Seasonal distribution of hepatitis in Sweden, 1931-68.

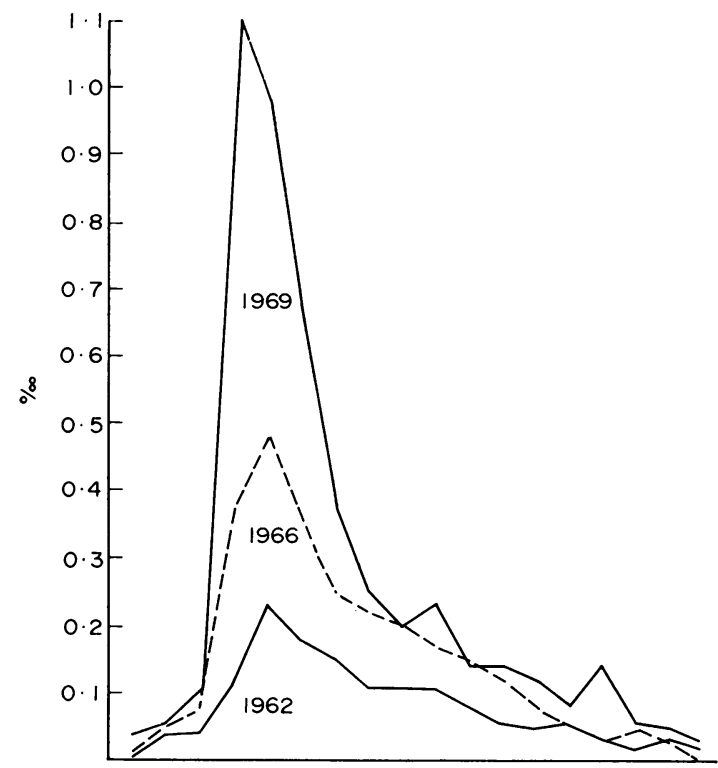

+ $\begin{array}{llllllllllllllllll}1 & 1 & 1 & 1 & 1 & 1 & 1 & 1 & 1 & 1 & 1 & 1 & 1 & 1 & 1 & 1 & 1 & 11\end{array}$

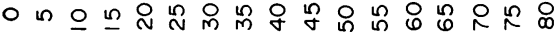

Age

FIg. 11. Age distribution of hepatitis in Sweden.

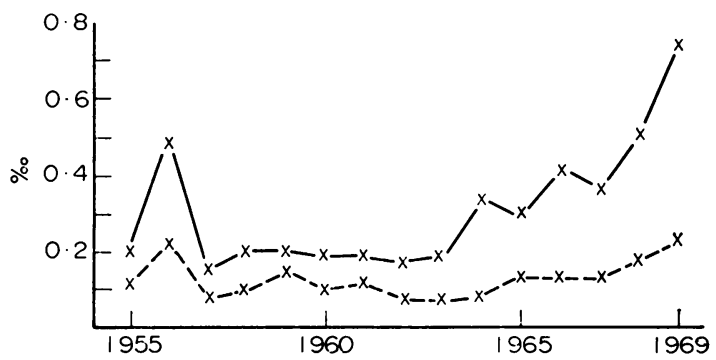

FIG. 12. Hepatitis morbidity in rural and urban areas in Sweden, 1955-69.x-x, Stockholm, Gothenburg and Malmö; x - - x, whole country excluding Stockholm, Gothenburg and Malmö.

Figure 12 illustrates the hepatitis morbidity in the three large cities in Sweden compared with the rest of the country. The figures of the urban areas are approximately twice as high as those of the rural areas. Both curves show a considerable increase during the last few years. Therefore, we are, in spite of the comparatively low hepatitis morbidity, not quite happy with the situation.

\section{References}

Berg, R., Ringertz, O. \& Espmark, Å. (1971) Australia antigen in hepatitis among Swedish track-finders. Acta pathologica et microbiologica Scandinavica, 79B, 423. 
Gille, G., Ringertz, O. \& Zetterberg, B. (1967) Serum hepatitis among Swedish track-finders. II. A clinical study. Acta medica Scandinavica, 182, 129.

RINGERTZ, O. \& Nyström, B. (1967) Viral hepatitis in connection with haemodialysis and kidney transplantation. Scandinavian Journal of Urology and Nephrology, 1, 192.

Ringek'z, O., Nyström, B. \& Ström, J. (1969) Clinical aspects on an outbreak of hepatitis among personnel in haemodialysis units. Scandinavian Journal of Infectious Diseases, 1, 51.

RINGERTZ, O. \& ZETTERBERG, B. (1967) Serum hepatitis among Swedish trackfinders. I. An epidemiological study. New England Journal of Medicine, 276, 540.

Roos, B. (1956) Hepatitepedemi spridd genom ostron. Svensk Läkartidning, 53, 989.

ZETTERBERG, B. (1964) Common source outbreaks of viral hepatitis. European Symposium on Viral Hepatitis, Prague. 